\title{
Hepatic rupture as the initial manifestation of HELLP syndrome
}

\author{
L Thiessen', J Shaw ${ }^{2}$ \\ ${ }^{1}$ University of Saskatchewan, Saskatoon, SK, Canada \\ ${ }^{2}$ Division of General Surgery, Royal University Hospital, Saskatoon, SK, Canada
}

Corresponding author: Lyndsey Thiessen (lbt714@mail.usask.ca)

\begin{abstract}
Summary: A 34-year-old normotensive nulliparous woman at 38 weeks' gestation developed sudden onset of severe right upper quadrant pain, fetal distress, hypotension, and a generalised seizure. At urgent Caesarean section, a ruptured subcapsular liver haematoma was found. A healthy infant was delivered. Haemostasis was obtained with a Pringle manoeuver and liver packing. Subsequent bloodwork diagnosed HELLP syndrome. Both the patient and the infant made a full recovery. This case demonstrates the effectiveness of prompt application of these surgical manoeuvres.
\end{abstract}

\section{Case}

A healthy 34-year-old nulliparous woman at 38 weeks' gestation presented to hospital with lower abdominal and hip pain. Assessment revealed she was in early labor, with a normal foetal heart rate and blood pressure of 128/82 mmHg. Two hours later she developed acute onset of severe right upper quadrant pain that was not responsive to morphine. The foetal heart rate had decreased to 66 beats per minute, and she was now hypotensive and tachycardic. She was taken to the operating room for an emergency Caesarean section for foetal distress and had a generalised seizure en route. At section, an intact uterus was found and a live female infant was delivered. There was a significant hemoperitoneum and an upper midline laparotomy was performed, identifying a ruptured right subcapsular liver hematoma. A Pringle manoeuver was performed and the liver was packed with sponges. Haemostasis was achieved after six minutes of Pringle clamping and packing. Intraoperative bloodwork showed a hemoglobin of $89 \mathrm{~g} / \mathrm{l}$, platelet count of $84 \times 10^{3} / \mu 1$, ALT of $526 \mathrm{u} / 1$, AST of $547 \mathrm{u} / \mathrm{l}$, and haptoglobin of $<0.10 \mathrm{~g} / 1$. A temporary abdominal closure system (Abtherra ${ }^{\circledR}$ ) was applied and the patient was taken to the intensive care unit (ICU) with a diagnosis of eclampsia and hemolysis, elevated liver enzymes, and low platelet syndrome (HELLP) complicated by spontaneous hepatic rupture.

The patient was taken back to the operating room 24 hours later for a relook laparotomy after resuscitation. No ongoing hepatic bleeding was identified. The packing was removed and abdomen closed. She returned to the ICU where she remained for several days before being transferred to the surgical ward. Postoperatively she developed an ileus that resolved with conservative management, as well as pulmonary oedema, atelectasis, and hypertension. She was discharged home on labetalol and nifedipine on post-delivery day 15 .

The patient has since been seen for follow up. Magnetic resonance imaging was subsequently performed, which revealed no underlying liver lesions. Both the patient and her infant are doing well.

\section{Discussion}

Preeclampsia is defined as blood pressure elevated above $140 / 90 \mathrm{mmHg}$ with proteinuria of $0.3 \mathrm{~g}$ over 24 hours after either 20 weeks of gestation or delivery. Preeclampsia has serious maternal and foetal health consequences, including potential development of eclampsia and HELLP. Eclampsia is diagnosed in women with preeclampsia who experience a seizure during pregnancy or postpartum that cannot be attributed to another identifiable cause.

HELLP is a life-threatening liver disorder defined by the following laboratory findings: LDH greater than $600 \mathrm{u} / 1$, AST greater than $70 \mathrm{u} / 1$, and platelets lesser than $150 \times 10^{3} / \mu 1 .^{1}$ Platelet counts may be used to grade HELLP, with $100-150$ $\mathrm{x} 10^{3} / \mu 1$ classified as mild, $50-99 \times 10^{3} / \mu 1$ as moderate, and fewer than $50 \times 10^{3} / \mu 1$ as severe. ${ }^{1}$

Preeclampsia complicates approximately $8 \%$ of pregnancies. ${ }^{2} 2-12 \%$ of pregnancies in which preeclampsia is diagnosed, or $0.2-0.6 \%$ of all pregnancies, are complicated by HELLP. ${ }^{1}$ Older, multiparous, and Caucasian women are more commonly affected. ${ }^{3}$ Numerous complications are associated with HELLP, including hepatic rupture, pulmonary oedema, cerebral oedema and haemorrhage, disseminated intravascular coagulation, acute renal failure, hepatorenal failure, placental abruption, acute respiratory distress syndrome, stroke, and sepsis. $^{2}$ 
The precipitating cause of HELLP is thought to originate from abnormal placental development, leading to ischemia and oxidative stress. ${ }^{4}$ The resulting inflammatory state preferentially targets the liver. The pathogenic process leading to hepatic rupture is thought to involve vascular endothelial injury, resulting from cytokine release and production of abnormal metabolic derivatives that activate the complement cascade. $^{5}$ Fibrin deposition in blood vessels with platelet activation and consumption causes thrombocytopenia and areas of hepatic haemorrhage and necrosis. This results in possible hematomas, capsule tears and intraperitoneal bleeding. Damage to the blood vessel intima, combined with fibrin deposition, may create shearing forces responsible for the observed hemolysis. ${ }^{6}$

Patients with preeclampsia or HELLP may present with symptoms including right upper quadrant or epigastric pain and tenderness, nausea, vomiting, malaise, headache, and oedema. ${ }^{1}$ Hypertension and proteinuria are often present. With hepatic rupture, hemodynamic instability, acute anaemia and foetal distress may additionally be observed. ${ }^{2,4}$ However, the presentation of hepatic rupture is inconsistent and patients may remain asymptomatic until sudden haemodynamic instability occurs. $^{3}$

Hepatic rupture is rare, reported in $0.53-2 \%$ of patients with preeclampsia or HELLP. ${ }^{4}$ One-third of patients rupture in the postpartum period with the majority occurring within 48 hours of delivery, though cases have been observed as late as six weeks. ${ }^{3}$ Maternal mortality rates from hepatic rupture are high, reported up to $50 \%{ }^{1}$ Perinatal mortality rates vary between $10-60 \%$ and are secondary to placental rupture, intrauterine asphyxia, or prematurity. ${ }^{1}$

Suspicion of hepatic rupture necessitates aggressive supportive care and an emergent laparotomy and delivery. ${ }^{1}$ Caesarean section should be performed immediately, as delivery is the definitive treatment for preeclampsia and HELLP. Numerous surgical options exist for managing hepatic rupture. Pressure packing, haematoma evacuation, and drainage are the most successful operative interventions, followed by ligation or embolization of the hepatic artery. If the above manoeuvers are unsuccessful partial hepatectomy, oversewing the laceration, fibrin gluing, and liver transplantation are other uncommon surgical interventions. ${ }^{1,3}$ Angiographic embolization may be an option in ruptured patients who are hemodynamically stable.

\section{Conclusion}

Hepatic rupture is a life-threatening complication of preeclampsia or HELLP syndrome requiring emergent surgical intervention and delivery. This case demonstrates that patients with hepatic rupture may not present with evident preeclampsia or HELLP. Therefore, hepatic rupture is an important differential diagnosis to consider in a gravid patient presenting with right upper quadrant pain and haemodynamic instability. Liver packing and haematoma evacuation and drainage remain the treatment of choice in management of hepatic rupture.

Conflict of Interest: None declared.

\section{REFERENCES}

1. Hay JE. Liver disease in pregnancy. Hepatology. Mar 2008;47(3):1067-76. Available from: doi:10.1002/hep.22130 [PMID: 18265410]

2. Vigil-De Garcia P, Ortega-Paz L. Pre-eclampsia/eclampsia and hepatic rupture. Int J Gynaecol Obstet. Sept 2012;118(3):186-9. doi: 10.1016/j.ijgo.2012.03.042 [PMID: 22717416]

3. Wilson SG, White AD, Young AL, Davies MH, Pollard SG. The management of the surgical complications of HELLP syndrome. Ann R Coll Surg Engl. Oct 2014; 96(7):512-6. doi: 10.1308/003 588414X13946184901362 [PMCID: 4473436]

4. Perronne L, Dohan A, Bazeries P, Guerrache Y, Fohein A, Rousset P, et al. Hepatic involvement in HELLP syndrome: an update with emphasis on imaging features. Abdom Imaging. Oct 2015 ; 40:2839-49. doi: 10.1007/s00261-015-0481-1 [PMID: 26099472]

5. Fang CJ, Richards A, Liszeweski MK, Kavanagh D, Atkinson JP. Advances in understanding of pathogenesis of aHUS and HELLP. Br J Haematol. Nov 2008; 143:336-48. doi: 10.1111/j.1365-2141.2008.07324.x [PMID: 18691170]

6. Reubinoff BE, Schenker JG. HELLP syndrome - a syndrome of hemolysis, elevated liver enzymes and low platelet count complicating preeclampsia/eclampsia. Int J Gynaecol Obstet. Oct 1991;36:95-102. [PMID: 1683323] 